\title{
Megaesophagus was Complicated with Billroth I Gastroduodenostomy in a Cat
}

\author{
Shunsuke SHIMAMURA ${ }^{1)}$, Miki SHIMIZU ${ }^{1)}$, Masayuki KOBAYASHI ${ }^{1)}$, Hidehiro HIRAO ${ }^{1)}$, Ryou TANAKA ${ }^{1)}$ and \\ Yoshihisa YAMANE ${ }^{1)}$ \\ ${ }^{1)}$ Department of Veterinary Surgery, Faculty of Agriculture, Tokyo University of Agriculture and Technology, 3-5-8 Saiwai-cho, Fuchu- \\ shi, Tokyo 183-8509, Japan
}

(Received 3 February 2005/Accepted 10 May 2005)

ABSTRACT. A seven-year-old, female, domestic short hair cat was presented with a history of chronic anorexia. Radiographic examination revealed a large space-occupying calcified mass in the abdominal cavity. The mass was located in pylorus and did not extend into the duodenum and surrounding tissues. Billroth I gastroduodenostomy was conducted to remove the mass. Histopathological examination of the mass showed a lymphoma. Although Recovery following the operation was excellent, the patient showed intermittent vomiting unrelated to feeding. Radiographical examination revealed a megaesophagus, which was assumed to be a complication of the Billroth I procedure, since the condition was not observed before the procedure.

KEY WORDS: billroth I, complication, megaesophagus.

Billroth I is one of the most common gastroduodenostomy procedures used to remove the pylorus. After removal of the affected part, the remaining parts of the stomach and duodenum are attached together using end-to-end anastomosis $[1,10,14]$. The procedure is also used to remove gastric neoplasm. Billroth II, which is quite similar to Billroth $\mathrm{I}$, is a well established procedure used for gastric resection. In Billroth II, however, restoration of the patency of the duodenum appears to be inadequate; thus Billroth I is more preferable procedure than Billroth II. Billroth I procedure, however, conteines the risk of suture line dehiscence and leakage caused by stress of gastrointestinal tract peristalsis due to the narrow gastroduodenal junction. In addition, technical difficulties in the procedure necessitate more extensive care for its adaptation. There are many studies reporting the use of Billroth I and Billroth II for gastric neoplasm and outflow obstruction in veterinary medicine. Almost of these studies reported good outcome, except for the presence of vomiting in some cases $[2,6,12,15]$. The present study describes the occurrence of megaesophagus following Billroth I procedure in a cat.

A seven-year-old domestic short hair cat, weighing 3.3 $\mathrm{kg}$, was presented to the Veterinary Teaching Hospital, Tokyo University of Agriculture and Technology, with complaints of depression and anorexia, without gastrointestinal symptoms. On the 1st day, abdominal palpation revealed a hard, mobile mass around upper quadrant; no swelling was observed on surface lymph nodes. Biochemical examinations revealed a moderate increase in glutamic oxaloacetic transaminase, lactate dehydrogenase. Radiographic examination of the ventral aspect of the abdomen revealed a mass a calcified center (Fig. 1). Thoracic radiographs showed no signs of metastasis. On the ultrasonography, a well defined mass containing a hyperechoic area approximately $4 \mathrm{~cm}$ in diameter was identified caudal to the stomach.

An exploratory laparotomy was performed to define the nature and extent of the lesion. The mass was seen occupying the upper quadrant of the abdomen and involved the pylorus area. However the mass was not seen infiltrating the duodenum, surrounding tissues, and regional lymph node. Billroth I was performed to remove the solitary mass in the pylorus, relieve the clinical signs and obtain tissues for pathological diagnosis. The schema of operation is illustrated in Fig. 2. The stomach and duodenum were separated and supported with umbilical tape. The mass, including a 2$\mathrm{cm}$ healthy tissue surrounding the mass, was dissected from the stomach and duodenum. The dissection left the gastric and duodenal lumens with different sizes. The antrum side was partly closed with a 2-layer suture; then, end-to-end anastomosis of the pyloric antrum with the duodenum was performed using a 2-layer suture (Fig. 3). Finally, after confirming that the common bile duct and pancreatic duct sustained no damage, a jejunostomy tube was inserted for nutritional support. Subsequent histopathologic examina-

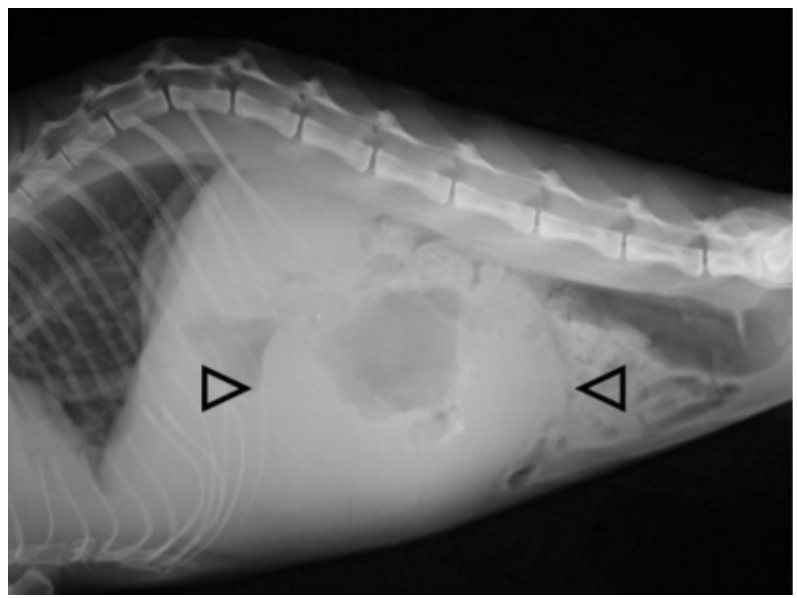

Fig. 1. Abdominal radiograph in a cat showing egg-size mass (black arrows). 


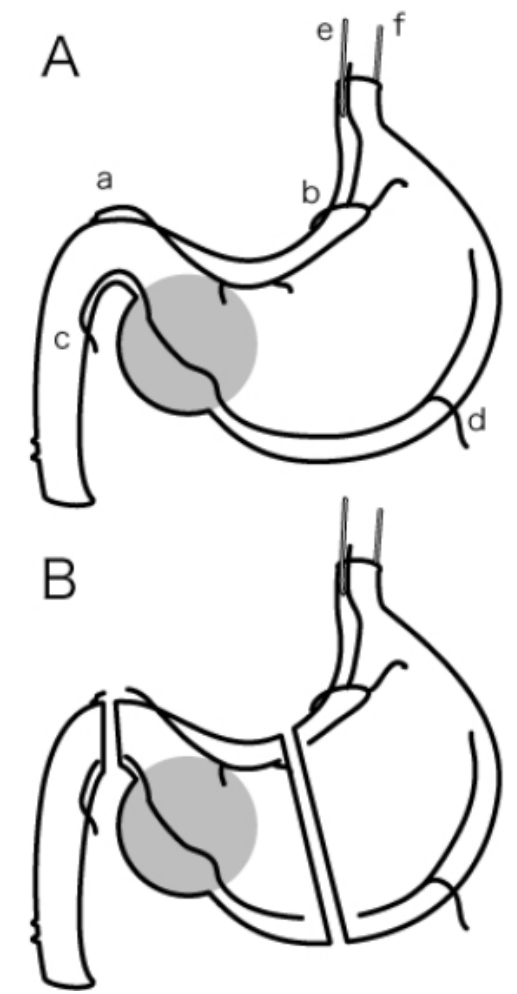

Fig. 2. Schematic representations of performing procedure of the Billroth I. Stomach was illustrated in pre-resection (A) and post-resection (B). a) right gastric artery b) left gastric artery c) right gastroepiploic artery d) left gastroepiploic artery e) ventral vagal trunk f) dorsal vagal trunk.

tion of the mass confirmed the diagnosis as lymphoma.

One day after the operation, jejunal feeding was started using a commercial enteral feeding solution (Clinical Care ${ }^{\circledR}$; Abbott Laboratories, Abbott Park, IL, U.S.A.) administered at a rate calculated to provide half of the daily caloric requirements. The volume of enteral formula was gradually increased to maintenance volume the following day. After observing the return of appetite on the 6th day, and confirming that there is no leakage in the gastroduodenal area through gastrography, oral feeding was started. When oral feeding became well tolerated, jejunal feeding was decreased gradually. On the 9th day postsurgery, the jejunostomy tube was removed and the cat was discharged from the hospital after confirmed the passage of radiopaque material from the stomach into the intestine. On the 22 nd day after operation, the patient was presented to the hospital with signs of intermittent vomiting unrelated to eating. Thoracic radiographs revealed a megaesophagus (Fig. 4). However, there was only a slight weight loss. The owner was instructed feed the cat in an upright position to take advantage of gravity drainage through a non-peristaltic esophagus. Thereafter, the cat's appetite returned to normal but

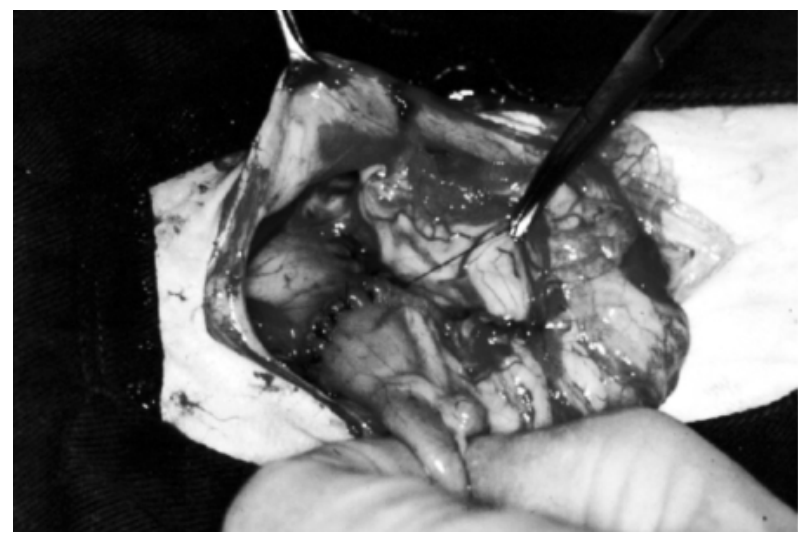

Fig. 3. Intraoperative view of the junctional area of stomach and duodenum in a cat. Anastomosis was performed using two-layer suture with 4-0 nylon.

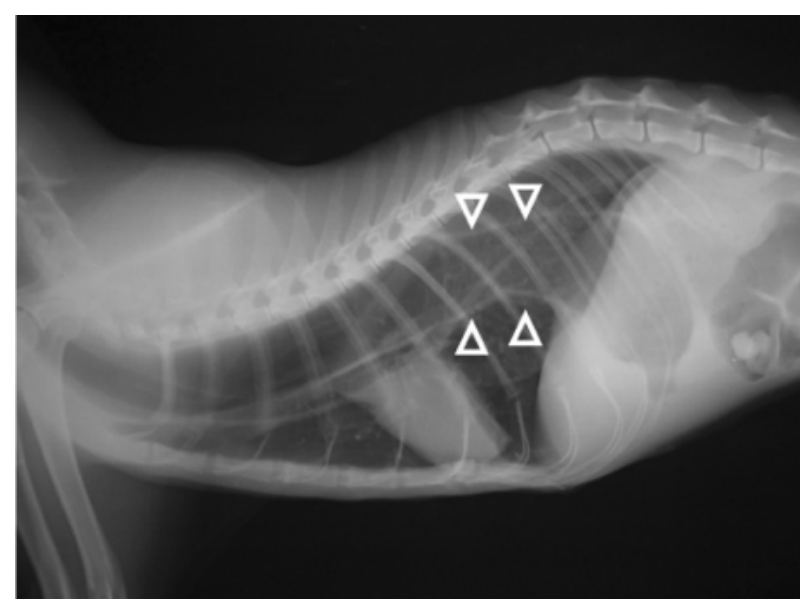

Fig. 4. Lateral radiograph showing the megaesophagus 22nd day after surgery in a cat. White arrows assigned the dilated esophagus.

chronic intermittent vomiting continued.

In human medicine, suture line dehiscence and leakage, pernicious anemia, and dumping syndrome were reported as complications of Billroth I procedure [9, 13]. However, in veterinary medicine, there has been no known report of complications using this procedure. Necrosis of the gastroduodenal junction caused by high-tension suture line and stress due to movement of gastrointestinal tract are thought to be major contributors in suture line leakage. In contrast with humans in standing position, gravitation stress in suture line is lesser in animal. In this case, leakage was not detected by gastrography after the operation. Dumping syndrome is symptoms caused by rapid drainage of the stomach with gastrectomy [3]. A decreased plasma volume is attributed to the efflux of fluid into the intestinal lumen causing concentration of blood into the mucosa and then extracelluar fluid transfer by increased osmotic gradient. Stimulation of the jejunal mucosa induces changes in multiple hormones 
and peptides (serotonin, substance $\mathrm{P}$, kinins, and gut glucagons). These vasoactive peptides cause vasomortor and intestinal symptoms [4]. Shortening the retention time of stomach induces acute hyperglycemia after feeding. This temporary hyperglycemia induces hypersecretion of insulin from the pancreatic islet beta-cells, resulting in hypoglycemia. In this case, the appearance of the above complications were monitored during the Billroth I procedure; however, none of these symptoms occurred. The body weight, though, kept on decreasing, although the cat maintained its appetite and received its daily caloric requirements. Blood glucose and cholesterol were examined before and after feeding high calorie diet in order to observe absorption of feed; however, significant changes were not observed. It is suggested that shortening the time of stay in the stomach will cause poor homogenization, digestion and absorption. Enteral feeding was, thus, given orally and the animal gradually recovered its body weight. Primary feeding of easily absorbable food to patient with pyloric antrum resection should be monitored carefully for possible occurrence of artificial dumping syndrome. Previous reports describe complication of lymphoma or thymoma with megaesophagus $[5,8]$. Although the present case was diagnosed as lymphoma, megaesophagus appears to be a complication of Billroth I since it was observed only after surgery. Megaesophagus can be caused by vagal afferent nerve dysfunction, as described in a previous report [7]. Thus, great care was observed in the present study to avoid injury to the vagal nerve. Esophagitis, which induces reflux to esophagus, is thought to be one factor which contributes to the occurrence of megaesophagus [16]. It is suggested that reflux to the esophagus induces disorder in digestion by losing large space of the stomach and this, together with the dysfunction of esophageal sphincters as affected by anesthesia, may be the cause of megaesophagus. In fact, anesthesia induces gastric reflux due to decrease the lower oesophageal pressure in dogs and cats [11]. Although chronic intermittent vomiting was observed in the cat on 8 months after surgery, the surgery was considered to be successful. Resection of pylorus area may induces gastrointestinal tract dysfunction, resulted in prolonged retention time of stomach, because the pacemaker of upper digestive tract is existed in pylorus area. Thus, metoclopramide, gastrointestinal stimulant, was treated, but vomiting was not improved. Megaesophagus can be treated by restoring the volume of stomach, if this condition is indeed caused by decreased stomach volume. It is difficult to know the primal cause of vomiting after occurrence of megaesophagus. Because, the both decreased stomach volume and megaesophagus has a possibility to induce vomiting. However, the dilated esophagus is thought as more reasonable inducer of vomiting, because, in this case, the vomiting that the cat presented was unrelated to eating.

The present report describes a complication of Billroth I procedure which was not described previously in veterinary medicine. A number of reports regarding complications of Billroth I procedure have been reported in human medicine. This study is the first known case reporting on the occurrence of megaesophagus following Billroth I procedure.

\section{REFERENCES}

1. Ahmadu-Suka, F., Withrow, S. J., Nelson, A. W., Husted, P. W., Gillette, E. L. and Whiteman, C. E. 1988. Vet. Surg. 17: 211-219.

2. Bellenger, C. R., Maddison, J. E., MacPherson, G. C. and Ilkiw, J. E. 1990. Aust. Vet. J. 67: 317-320.

3. Carvajal, S .H. and Mulvihill, S. J. 1987. Gastroenterol. Clin. North Am. 23: 261-279.

4. Cooperman, A. M. 1981. Surg. Ann. 13: 139-161.

5. Day, M. J. 1997. J. Small Anim. Pract. 38: 393-403.

6. Godshalk, C. P., Roush, J. K., Fingland, R. B., Sikkema, D. and Vorhies, M. W. 1992. J. Am. Vet. Med. Assoc. 201: 17341736.

7. Holland, C. T., Satchell, P. M. and Farrow, B. R. 1996. Am. J. Vet. Rec. 57: 906-913.

8. Kline, M. M. 1980. Digest. Dis. Sci. 25: 311-313.

9. Kyzer, S., Binyamini, Y., Melki, Y., Ohana, G., Koren, R., Chaimoff, C. and Wolloch, Y. 1997. World J. Surg. 21: 763766.

10. Matthiesen, D. T. 1993. pp. 615-622. In: Textbook of Small Animal Surgery, 2nd, ed. W.B. Saunders, Philadelphia.

11. Pearson, H., Darke, P. G., Gibbs, C., Kelly, D. F. and Orr, C. M. 1978. J. Small Anim. Pract. 19: 507-519.

12. Runk, A., Kyles, A. E. and Downs, M. O. 1999. J. Am. Anim. Hosp. Assoc. 35: 52-55.

13. Thomas, W. E., Thompson, M. H. and Williamson, R. C. 1982. Ann. Surg. 195: 189-195.

14. Walter, M. C. and Matthiesen, D. T. 1989. Prob. Vet. Med. 1: 196-214

15. Walter, M. C., Matthiesen, D. T. and Stone, E. A. 1985. J. A. Vet. Med. Assoc. 187: 909-914.

16. Washabau, R. J. 2000. pp. 1143-1154. In: Textbook of the Veterinary Internal Medicine, 5th ed., W.B. Saunders, Philadelphia. 